\title{
Recovery From Cervical Dystonia With Topiramate
}

\author{
Anu Putkonen
}

\begin{abstract}
Primary cervical dystonia (CD), spasmodic torticollis, is the most common focal dystonia. In the US between 60,000 and 90,000 people have been estimated to suffer from this painful disorder with rare recovery and high psychiatric co-morbidity. The therapy of CD has been symptomatic. The primary treatment, repeated chemodenervation of cervical muscles with botulinum toxin injections, is expensive and not available to the majority of persons with $\mathrm{CD}$. Neither controlled studies of oral pharmacotherapy of dystonia, nor reports of sustained full remission were published. Thus, it was unclear if any effective and safe oral pharmacotherapy for $\mathrm{CD}$ exists, and if it is possible to remain asymptomatic. I report on a 48 -year-old woman who has been symptom-free during nearly 4 years treatment with topiramate, after previously suffering from familial CD during 29 years. Her left-sided lateral postural and rotating dystonia increased in stressful situations and during physical exercise, and had not responded to any drugs including Botox injections. Topiramate (150 $\mathrm{mg}$ /day) was administered for her schizo-affective disorder in 2011, after 11 months of treatment with clozapine $(150 \mathrm{mg} /$ day $)$ in a forensic psychiatric hospital. Postural and rotating symptoms subsided within 2 months, and muscular hypertrophy, pain, dysphagia, and daily headache recovered. The author examined her lifetime health care referrals and documented her symptoms in the hospital during 6 years. Informed consent was obtained. CD is associated with reduction of gamma-aminobutyric acidergic (GABAergic) neurons in the basal ganglia. Topiramate, a sulfamate-substituted monosaccharide, modulates both GABAergic and glutaminergic neurotransmission. It has been used since the 90s for epilepsy and many neurological and psychiatric disorders, on the basis of randomized controlled trials. My case study demonstrates that some patients recover from CD during treatment with topiramate, and remain asymptomatic for years. This is in accordance with the pharmacodynamic mechanisms of topiramate, the pathophysiology of involuntary movements, and the clinical evidence of other hyperkinetic movement disorders with similar pathophysiology. It would be important to conduct randomized controlled studies of topiramate in $\mathrm{CD}$, and to define the subgroups
\end{abstract}

Manuscript accepted for publication June 30, 2015

Niuvanniemi Hospital, Department of Forensic Psychiatry, University of Eastern Finland, Kuopio, Finland. Email: putkonen@niuva.fi

doi: http://dx.doi.org/10.14740/jmc2088w who may benefit from it.

Keywords: Cervical dystonia; Topiramate; Recovery; Movement disorder; Case report

\section{Introduction}

Primary cervical dystonia (CD) is defined as sustained involuntary muscle contractions that cause twisting, repetitive and patterned movements, or abnormal postures of the neck [1]. About half of the patients also suffer from head tremor, and for $75 \%$ the chronic dystonic activity causes painful cervical muscular hypertrophy [2]. The prevalence of primary CD is unclear as $\mathrm{CD}$ is often not recognized by physicians, and no validated diagnostic criteria existed. The minimum incidence estimation was 8-12 cases per million person years [3]. Women are affected $1.5-1.9$ times more often than men, and the majority of these disorders begin in the middle age [3]. The estimated number of persons having CD in the US is between 60,000 and 90,000 [4]. During the first years after onset, some patients have periods with fewer symptoms but recovery is rare [2].

The treatment of CD has been symptomatic, and aims to relieve the involuntary movements, prevent contractures, decrease pain and embarrassment, and to improve function [1, 5]. The primary treatment, chemodenervation of cervical muscles with botulinum toxin injections every 3 - 4 weeks, has temporarily relieved some symptoms of $75 \%$ of patients [1]. Also intrathecal baclofen and deep brain stimulation have been effective in randomized controlled trials. However, these invasive treatments are expensive and generally not available or possible to the greater majority of those who suffer from $\mathrm{CD}$, e.g., in developing countries.

The efficacy and tolerability of oral pharmacotherapy for $\mathrm{CD}$ is poorly documented. No controlled data are available, and most published recommendations are based on empirical observations [1]. Anticholinergic drugs, benzodiazepines and clozapine have decreased some symptoms in some cases [1], and attacks of kinesigenic paroxysmal dystonia can sometimes be controlled with anticonvulsants while non-kinesigenic symptoms are less responsive to pharmacotherapy [6]. However, neither controlled data of oral pharmacotherapy of $\mathrm{CD}$, 
nor reports of sustained full remission were published [1]. Thus, it was unclear if any effective and safe oral pharmacotherapy for CD exists, and if it is possible to cure all symptoms of $\mathrm{CD}$ with an oral pharmacotherapy.

\section{Case Report}

Herein, I report of the recovery of a 48 -year-old woman who has now been symptom-free nearly 4 years during treatment with topiramate, after previously suffering from rotational lateral CD during 29 years. The patient's symptoms begun at the age of 12 with head tremor. Her lateral, left-sided rotational dystonia began when she was 15 , and increased in both social and stressful situations, and during physical activity. Her grandmother had suffered from a similar dystonia. In her twenties, the patient also suffered from social phobia, anxiety, borderline and depressive symptoms and alcohol dependence, while her dystonia was misdiagnosed as a panic disorder, and treated with amitriptylene/chlordiazepoxid and benzodiazepines. She used citalopram (up to $45 \mathrm{mg}$ ) and moclobemide (150 mg) temporarily but suffered from restlessness, agitation and hypomanic symptoms during them. At the age of 34, a diagnosis of CD in the Clinic of Neurology of the Central Hospital was a relief to the patient. However, her symptoms did not respond significantly to any pharmacotherapy, including botulinum toxin injections which she received regularly during 1 year. The patient achieved some relief only from benzodiazepines and alcohol.

At the age of 42 her lifetime health care documents were reviewed in a forensic psychiatric examination, and the patient was diagnosed with schizo-affective disorder, alcohol dependence and benzodiazepine dependence. MRI study revealed no structural brain abnormalities. The left-handed patient suffered from postural and rotating left-sided CD and a slight cervical tremor; cervical muscular hypertrophy and pain, and dysphagia due to the distortion of the esophagus. She also had daily headache, and both paranoid and depressive delusions. In the forensic psychiatric hospital administration of olanzapine resulted in metabolic syndrome. Ziprasidone could not be used because of akathisia, increased tremor and an urge to twist her body. These symptoms increased in the evenings, as well as the symptoms of CD. Clozapine (150 mg/day), administered since September of 2010, relieved paranoid symptoms. Topiramate (up to $150 \mathrm{mg} /$ day) was administrated as an adjuvant since $\mathrm{Au}-$ gust of 2011, to replace lamotrigin. Unexpectedly, the rotating and postural symptoms subsequently disappeared within less than 2 months during the increase of the dose up to 150 $\mathrm{mg}$ /day, and gradually also the muscular hypertrophy, cervical pain, daily headache, tremor and dysphagia recovered. During some weeks the patient still felt while swallowing as if there now was a curve in her esophagus.

The patient has now been symptom-free from the dystonia after nearly 4 years of topiramate $150 \mathrm{mg}$, clozapine $175 \mathrm{mg}$, fluoxetine $15 \mathrm{mg}$ and bisoprolol $2.5 \mathrm{mg}$ per day. In stressful situations, she still has very slight tremor in hands, but no abnormal movements or positions. Gradually, also her delusions and social fears have subsided. The patient is very happy of her recovery. She wishes that an article of it would encourage research and help others who suffer from this painful and dis- tressing disorder.

\section{Discussion}

This case demonstrated that some patients recover from CD and remain asymptomatic for years during treatment with topiramate. It was possible to follow the previous development of the symptoms of CD from the detailed health care data collected for the profound mental state examination in a university clinic. The author has examined the patient and discussed with her regularly since her admission to a forensic psychiatric hospital, and documented her symptoms during 6 years. No postural or rotating symptoms have relapsed this far.

The role of clozapine in this recovery was unclear. A small non-controlled pilot study $(\mathrm{n}=10)$ reported that clozapine decreased clonic symptoms of CD but had no effect on torticollis [7]. In the present case clozapine $(150 \mathrm{mg} /$ day $)$ was administered without any effect on CD during 11 months before the administration of topiramate. In theory, it may have had some adjunctive effect on the clonic component. The small dose of fluoxetine (15 mg/day) administered 2 months before topiramate may have increased the concentration of clozapine and topiramate in the serum. However, fluoxetine was not likely to be involved in the improvement of CD after the administration of topiramate. The patient had previously used other SSRIs without any relief of the CD. It had increased restlessness, and $45 \mathrm{mg}$ citalopram/day had induced manic symptoms.

The pathophysiology of involuntary movements in dystonia and chorea, essential tremor, myoclonus and restless legs syndrome has been associated with a reduction of gamma-aminobutyric acidergic (GABAergic) neurons in the basal ganglia [7]. The reduced output results in thalamo-cortical system disinhibition, which releases the cortical motor areas generating the normally suppressed movements [7]. Changes in the patterning or synchronization of basal ganglia output, as well as dopaminergic system dysfunction in the basal ganglia, may be involved in some hyperkinetic disorders, and also in schizophrenia [7].

Topiramate, a sulfamate-substituted monosaccharide, modulates both GABAergic and glutamatergic neurotransmission, as well as voltage-gated ion channels or intracellular signaling pathways [7]. The efficacy and tolerability of topiramate has been reported with several randomized controlled studies for many neurological and psychiatric disorders, e.g. for some epileptic disorders, Lennox-Gastaut syndrome, and migraine, essential tremor, vascular generalized chorea, and as an adjunct to clozapine in treatment resistant schizophrenia [7-9]. It has been used since 1990s for some forms of epilepsy and for migraine. There are reports of improvement of cluster headache, schizo-affective disorders, bipolar disorder, bulimia and neuropathic pain syndromes during treatment of topiramate. Clinical evidence suggests the usefulness of topiramate also in some hyperkinetic disorders, i.e. essential tremor, chorea, restless legs syndrome and myoclonus. It has also reduced symptoms in single cases of spinal myoclonus and Tourette's syndrome [7]. The only case report of improvement of dystonia demonstrated a marked improvement of the symptoms 
of a primary cervico-trunco-brachial segmental dystonia of a 46-year-old man whose CD had already responded to botulinum injections during 11 years. During 2 months treatment with topiramate (up to $200 \mathrm{mg}$ /day) his trunk and upper limb dystonic component, trunk flexion and action-induced wrist extension improved considerably. The dystonic symptoms deteriorated after discontinuation of topiramate because of angle closure glaucoma, weight loss and word-finding difficulty [10].

\section{Conclusion}

This case demonstrates that some patients recover from CD during treatment with topiramate, and remain asymptomatic for years. This is in accordance with the pharmacodynamic mechanisms of topiramate, the pathophysiological mechanisms of involuntary movements [7], and with the clinical evidence of topiramate in some other hyperkinetic movement disorders with similar pathophysiology [7]. It would be important to conduct randomized controlled studies of topiramate in CD, and to define the subgroups who may benefit from it.

\section{Conflict of Interest}

None.

\section{Funding}

This study was supported by Niuvanniemi Hospital, Finland. The funder was not involved in the conduct of the study or in the collection, management, analysis or interpretation of the data.

\section{References}

1. Jankovic J. Treatment of hyperkinetic movement disorders. Lancet Neurol. 2009;8(9):844-856.

2. Tarsy D, Simon DK. Dystonia. N Engl J Med. 2006;355(8):818-829.

3. Defazio G, Jankovic J, Giel JL, Papapetropoulos1 S. Descriptive epidemiology of cervical dystonia. Tremor Other Hyperkinet Mov (N Y). 2013;3:pii tre-03-193-4374-2.

4. Velickovic M, Benabou R, Brin MF. Cervical dystonia pathophysiology and treatment options. Drugs. 2001;61(13):1921-1943.

5. Albanese A, Barnes MP, Bhatia KP, Fernandez-Alvarez E, Filippini G, Gasser T, Krauss JK, et al. A systematic review on the diagnosis and treatment of primary (idiopathic) dystonia and dystonia plus syndromes: report of an EFNS/MDS-ES Task Force. Eur J Neurol. 2006;13(5):433-444.

6. Lotze T, Jankovic J. Paroxysmal kinesigenic dyskinesias. Semin Pediatr Neurol. 2003;10(1):68-79.

7. Siniscalchi A, Gallelli L, Giofre C, De Sarro G. What's the role of topiramate in the management of patients with hyperkinetic movement disorders? Pharmacol Rep. 2012;64(1):24-30.

8. Tiihonen J, Halonen P, Wahlbeck K, Repo-Tiihonen E, Hyvarinen S, Eronen M, Putkonen H, et al. Topiramate add-on in treatment-resistant schizophrenia: a randomized, double-blind, placebo-controlled, crossover trial. J Clin Psychiatry. 2005;66(8):1012-1015.

9. Connor GS. A double-blind placebo-controlled trial of topiramate treatment for essential tremor. Neurology. 2002;59(1):132-134.

10. Papapetropoulos S, Singer C. Improvement of cervicotrunco-brachial segmental dystonia with topiramate. J Neurol. 2006;253(4):535-536. 